\title{
THE IMPACT OF A WELL-ORGANIZED STROKE NETWORK ON \\ ACUTE STROKE MANAGEMENT: \\ AN INITIAL DATA OF ACUTE STROKE ENDOVASCULAR THERAPY
}

\section{MLPCARE}

MEDICALPARK $\mid$ Ii $V_{\text {HOSPITAL }} \mid$ VM MEDICALPARK

Koyuncu, $B^{1}$, Bajrami, $A^{2}$, Pourmohammed R ${ }^{2}$, Akil, E ${ }^{3}$, Onalan, $A^{1}$, Nar Senol, $P^{1}$, Geyik, $^{1}$, Tolun, ${ }^{1}$, Krespi, $Y^{1}$

${ }^{1}$ Istanbul Istinye University Liv Hospital, Department of Neurology, Istanbul

${ }_{2}^{2}$ Istanbul Aydin University, Florya Medicalpark Hospital, Department of Neurology, Istanbul

${ }_{3}$ Dicle University, Faculty of Medicine, Department of Neurology, Diyarbakir

\section{BACKGROUND:}

Effective implementation of acute stroke care is strictly related to institutional and geographical limitations. Stroke Network aims to minimize such barriers to secure timely and effective acute treatment. We reviewed the results of the first 3 months experience.

\section{METHODS:}

Brain Angiography and Stroke Centre (BASC) Network includes three comprehensive stroke centres (CSC) distributed in different regions of Istanbul. Angio suite was established on October and Rapid software was implanted on December 2017. The BASC data (OctoberDecember 2017) is prospectively collected according to the SITS database. The results of endovascular treatment were reviewed.

\section{RESULTS:}

A total of 186 acute stroke patients (mean age 68 years (35-95)) were admitted. Seventy-four patients received revascularization therapy (27\% only IVT, $46 \%$ only mechanical thrombectomy (EVT), $27 \%$ Bridging therapy). Mean NIHSS at onset was 9. The main demographic and clinical features are shown in Table 1. A total of 54 patients received EVT and among these 2 patients remained as intention-to-treat. Revascularization timeframes are shown in Graphic 1. Stentretriever was used in a total of 45 patients and solely penumbra aspiration technique was used in only 3 patients. Out of $31(16,7 \%)$ patients with target M1 segment of MCA occlusion, 27 (51,9\%) received acute revascularization therapy. A total of 10 patients (19\%) underwent EVT following $>6 \mathrm{~h}$ of onset. Occluded arteries could be opened with $\mathrm{TICl}$ flow $\geq 2 \mathrm{~b}$ in 125 cases (91.2\%). In 121 cases (88.32\%) successful revascularization was achieved within one or 2 passes and $\mathrm{TICl} 2 \mathrm{~b}-3$ following only one pass was achieved in a total of 21 (41\%) patients. At discharge favourable(mRS 0-2) outcome rate was $46 \%$. In-hospital mortality rate was $4.3 \%$. Only 1 symptomatic haemorrhage occurred $(1,9 \%)$.

Graphic 1: Revascularization timeframes in EVT patients (median).

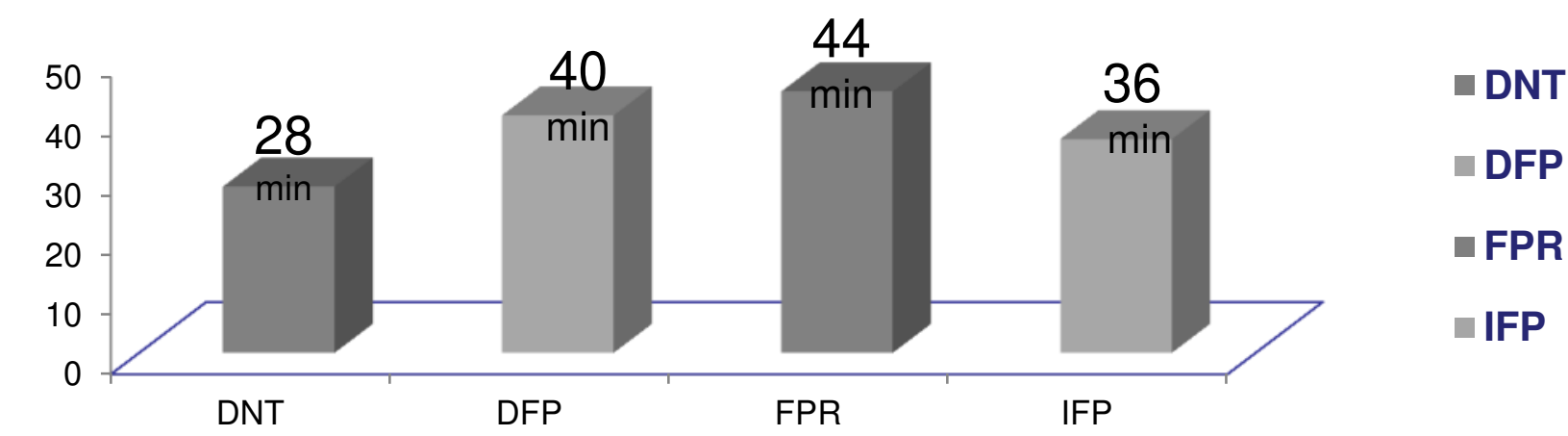

DNT: Door to needle time; DFP: Door to femoral puncture time; FPR: Femoral puntucre to revascularızation tıme; IFP: Imaging to femoral puncutre tıme.

Graphic 2: Patients' NIHSS values (median)

\section{CONCLUSION:}

Networking facilitated the access to IVT and/or EVT treatment in eligible patients. Our geographic setting, established interaction between centres and early access to multimodal, automatized, penumbral imaging modalities constitute the major contributing factors in obtaining the present results which indicate improved outcomes and benchmark indicators.

Table 1. Characteristic features of the study group including demographic and clinical data.

\begin{tabular}{|lc|}
\hline & $\mathbf{N}(\%)$ \\
\hline Ischemic Stroke & $147(95)$ \\
TIA & $10(5.4)$ \\
Gender- MALE & $106(57)$ \\
\hline 80 y & $39(21)$ \\
RT & $74(40)$ \\
IVT & $20(27)$ \\
BT (IVT + EVT) & $20(27)$ \\
EVT & $34(45)$ \\
Ant. Circulation & $134(72)$ \\
Vascular Pathology & $56(100)$ \\
& \\
M1 & \\
ICA T-Ocl. & $31(17,4)$ \\
ICA prox. & $17(9,5)$ \\
M2-M3 & $16(8,9)$ \\
ICA-M1 (tandem) & $12(6,7)$ \\
VA & $6(3.4)$ \\
BA & $9(5)$ \\
PCA & $4(2.2)$ \\
A2 & $4(2,2)$ \\
Unwitnessed or wake-up stroke & $1(0,5)$ \\
AF & $35(19)$ \\
Onset NIHS $>=10$ & $69(36)$ \\
ODT $(\%$ Total) & $77(42)$ \\
ODT < 4.5 h & \\
ODT <6 h & 52 \\
ODT $(\%$ EVT) & 61 \\
ODT < 4.5 h & \\
ODT < 6 h & 79 \\
\hline
\end{tabular}

TiA: Transient Ischemic atack; RT: Revascularization Therapy; IVT: Intravenous Thrombolysis; BT: Bridging Therapy; EVT: Endovascular Treatment; AF: Atrial Fibrilation; ODT: Onset to door time

Table 2. Overall complications and prognosis features

\begin{tabular}{|lccc|}
\hline \multicolumn{1}{|c}{$\%$} & Total & RT & EVT \\
\hline SITS - MOST & 0,7 & 1,3 & 1,6 \\
In Hospital Mortality & 4,2 & 6,3 & 7,9 \\
Discharge mRS 0-2 & 49,2 & 44,3 & 44,9 \\
3. month mortality & 16 & 20 & 20,3 \\
3. month mRS 0-2 & 44,6 & 36,3 & 32,8 \\
\hline
\end{tabular}

Graphic 3. Relationship between nr of passes, stentretriever and $\mathrm{TICI}$
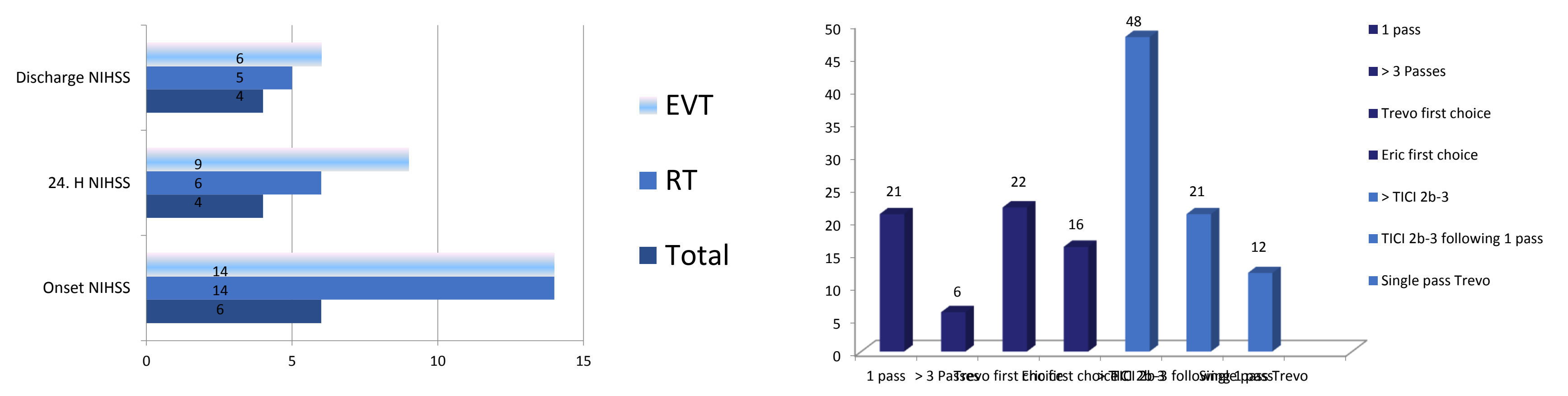